\title{
Heat transfer at nanometric scales described by extended irreversible thermodynamics
}

\author{
Hatim Machrafi ${ }^{1 *}$ \\ ${ }^{1}$ Thermodynamics of Irreversible Phenomena, \\ University of Liège, Liège, Belgium \\ *Email address for correspondence: H.Machrafi@ulg.ac.be \\ Communicated by Vito Antonio Cimmelli and David Jou \\ Received on December 9, 2014. Accepted on May 7, 2015.
}

\begin{abstract}
The purpose of this work is to present a study on heat conduction in systems that are composed out of spherical and cylindrical micro- and nanoparticles dispersed in a bulk matrix. Special emphasis is put on the dependence of the effective heat conductivity on various selected parameters as particle size and also its shape, surface specularity and density, including particle-matrix interaction. The heat transfer at nanometric scales is modelled using extended irreversible thermodynamics, whose main feature is to elevate the heat flux vector to the status of independent variable. The model is illustrated by a Copper-Silicium (Cu-Si) system. It is shown that all the investigated parameters have a considerable influence, the particle size being especially useful to either increase or decrease the effective thermal conductivity.

Keywords: nanometric heat transfer, thermal conductivity, extended irreversible thermodynamics, spherical and cylindrical nanocomposites.

AMS subject classification: 35Q79, 80A20, 82C35, 82D80.
\end{abstract}

\section{Introduction}

We investigate in this work some particular properties of heat conduction at nanometric scale. We are interested in systems where spherical or cylindrical nanoparticles are embedded in a host matrix, i.e. nanocomposites. Nanocomposites know a huge variety of applications, such as heat conduction enhancement in polyesters [1] or energy storage systems [2], to mention a few. Studies on the thermal conduction in porous media have been of great interest due to their applications in, for instance, material design, geophysical exploration, biological and medical engineering [3,4]. The change in thermal conductivity has also been exploited to increase the figure of merit $\mathrm{ZT}$ of thermoelectric materials which behaves as the inverse of 


\section{H. Machrafi}

the heat conductivity [5]. The nanocomposite is generally made out of a homogeneous matrix in which nanoparticles are dispersed. For particles with diameters of the same order of magnitude or smaller than the phonon mean free path, the Fourier theory, based on the classical approach of thermodynamics, is not able to predict the heat flux of thermal interactions between the matrix and the nanoparticles. Therefore, we propose to investigate the problem of heat conduction in nanocomposites by a more sophisticated thermodynamic formalism, namely Extended Irreversible Thermodynamics (EIT) [6]. In this approach, the heat flux is elevated to the status of independent variable at the same footing as the temperature. EIT has been successfully applied to transient heat transport in nanofilms in a previous paper [7]. The main problem investigated in the present work is the determination of the effective heat conductivity for nanocomposites with spherical and cylindrical embedded nanoparticles. We will make use of the effective-medium approach $[8,9]$ which provides a process of homogenization of the heterogeneous medium formed by the matrix and the particles. The basic formula for the effective heat conductivity coefficient $\lambda^{e f f, s}$ for spherical nanoparticles is Maxwell's relation

$$
\lambda^{e f f, s}=\lambda_{m}^{s} \frac{2 \lambda_{m}^{s}+\left(1+2 \alpha^{s}\right) \lambda_{p}^{s}+2 \varphi\left(\left(1-\alpha^{s}\right) \lambda_{p}^{s}-\lambda_{m}^{s}\right)}{2 \lambda_{m}^{s}+\left(1+2 \alpha^{s}\right) \lambda_{p}^{s}-\varphi\left(\left(1-\alpha^{s}\right) \lambda_{p}^{s}-\lambda_{m}^{s}\right)} .
$$

The effective heat conductivity for cylindrical nanoparticles $\lambda^{e f f, c}$ is given by $[8]$

$$
\lambda^{e f f, c}=\lambda_{m}^{c} \frac{3\left(\frac{\lambda_{r}^{e f f, c}}{\lambda_{m}^{c}}+1\right)+\varphi\left(2\left(\frac{\lambda_{r}^{e f f, c}}{\lambda_{m}^{c}}-1\right)+\left(\frac{\lambda_{r}^{e f f, c}}{\lambda_{m}^{c}}+1\right)\left(\frac{\lambda_{L}^{e f f, c}}{\lambda_{m}^{c}}-1\right)\right)}{3\left(\frac{\lambda_{r}^{e f f, c}}{\lambda_{m}^{c}}+1\right)-2 \varphi\left(\frac{\lambda_{r}^{e f f}, c}{\lambda_{m}^{c}}-1\right)},
$$

with $\lambda_{r}^{e f f, c}$ the radial component and $\lambda_{L}^{e f f, c}$ the longitudinal component of the cylindrical nanoparticles:

$$
\begin{gathered}
\lambda_{r}^{e f f, c}=\lambda_{m}^{c} \frac{\lambda_{m}^{c}+\left(1+\alpha^{c}\right) \lambda_{p}^{c}+\varphi\left(\left(1-\alpha^{c}\right) \lambda_{p}^{c}-\lambda_{m}^{c}\right)}{\lambda_{m}^{c}+\left(1+\alpha^{c}\right) \lambda_{p}^{c}-\varphi\left(\left(1-\alpha^{c}\right) \lambda_{p}^{c}-\lambda_{m}^{c}\right)}, \\
\lambda_{L}^{e f f, c}=(1-\varphi) \lambda_{m}^{c}+\varphi \lambda_{p}^{c} .
\end{gathered}
$$

In Equations (2)-(4), $\lambda_{m}^{s}$ and $\lambda_{p}^{s}$ stand for the heat conductivities of the matrix and the suspended particles, respectively, in the case of spherical 


\section{Nanoscale heat transfer}

nanoparticles, while $\lambda_{m}^{c}$ and $\lambda_{p}^{c}$ designate those in the case of cylindrical nanoparticles. Note that, as we will see later on, that also the heat conductivity of the matrix is influenced by the shape of the nanoparticles. Also, $\varphi$ is the volume fraction of the particles and $\alpha^{s}$ and $\alpha^{c}$ are dimensionless parameters, describing the thermal resistance due to particle-matrix interaction for spherical and cylindrical nanoparticles, respectively:

$$
\alpha^{s}=\lambda_{m}^{s} R / \ell_{p, s}
$$

$$
\alpha^{c}=\lambda_{m}^{c} R / \ell_{p, s}
$$

The quantity $R$ is the thermal boundary resistance coefficient given by [9]

$$
R=\frac{4}{C_{m} v_{m}}+\frac{4}{C_{p} v_{p}},
$$

while $\ell_{p, s}$ is the so-called "specular" characteristic length defined by

$$
\ell_{p, s}=\ell_{p} \frac{1+s}{1-s}
$$

with $\ell_{p}$ the characteristic length of the (spherical or cylindrical) nanoparticle. The symbols $C_{m}, v_{m}, C_{p}$ and $v_{p}$ in Equation (7) stand for the specific heat capacities and group velocities of the bulk matrix and the particles, respectively. In Equation (8), the symbol $s(0 \leq s \leq 1)$ denotes the surface specularity of the nanoparticles, expressing the probability of specular scattering of phonons on the particle-matrix interface. For $s=0$, the surface is called diffuse, meaning that the direction of phonons after impact is independent of the direction of the impacting phonons, in which case $\ell_{p, s} \equiv \ell_{p}$. For $s \rightarrow 1$, we have a surface on which the impacting phonons influence the direction of the outcoming phonons and the surface is said to be perfectly specular. Note that the result (5)-(6) was established in the case of diffusive surfaces [10]. The characteristic length $\ell_{p}$ is given by

$$
\ell_{p}=r_{p},
$$

in the case of spherical nanoparticles, where $r_{p}$ is the particle radius, and by

$$
\ell_{p}=\sqrt{\frac{1}{\frac{1}{r_{p}^{2}}+\frac{1}{L^{2}}}},
$$




\section{H. Machrafi}

in the case of cylindrical nanoparticles, where $L$ is the cylinder longitudinal length. Of course, in case $L>>r_{p}$, we would have $\ell_{p}=r_{p}$ for the cylindrical nanoparticle.

An expression for $\lambda_{m}^{s}$ and $\lambda_{m}^{c}$ is established in the framework of the effective-medium approach [8,9], while $\lambda_{p}^{s}$ and $\lambda_{p}^{c}$ will be derived from EIT. In the next section, we discuss briefly the principles of EIT, followed by the development of expressions for the matrix and particle thermal conductivities for both spherical and cylindrical nanoparticles. The model will be applied to a Copper-Silicium $(\mathrm{Cu}-\mathrm{Si})$ nanocomposite with comparisons to other models.

\section{Thermal conductivity by extended irreversible thermodynam- ics}

\subsection{Principles of extended irreversible thermodynamics}

In this subsection, we recall briefly the background of EIT. More details can be found in $[6,11]$. The principle idea behind EIT is to elevate the dissipative fluxes, as the fluxes of mass, energy and momentum to the status of independent variables at the same level as the classical variable like mass, energy or momentum. As a consequence, the space $\mathbf{V}$ of state variables will be formed by the union of the (slow and conserved) classical variables $\mathbf{C}$ and the (fast and non-conserved) flux variables $\mathbf{F}$ so that $\mathbf{V}=\mathbf{C} \bigcup \mathbf{F}$. In this work, we consider heat conduction in a rigid body at rest. In the problem of a rigid heat conductor, the only relevant conserved variable is the internal energy $e$ (or the temperature $T$ ) whereas the energy flux (here, the heat flux vector $\mathbf{q}$ ) is the non-conserved flux variable so that the space of state variables is $\mathbf{V}=\mathbf{V}(e, \mathbf{q})$. In more complex materials like in nanomaterials, fluxes of higher order should be introduced as shown later on. The corner stone of EIT is to assume the existence of an entropy function $\eta(\mathbf{V})$, depending on the whole set $\mathbf{V}$ of variables: here $\eta=\eta(e, \mathbf{q})$, or in terms of time $(t)$ derivatives,

$$
\partial_{t} \eta=\frac{\partial \eta}{\partial e} d_{t} e+\frac{\partial \eta}{\partial \mathbf{q}} \cdot d_{t} \mathbf{q}
$$

wherein $e$ and $\eta$ are measured per unit volume and a dot stands for the scalar product. The symbol $d_{t}$ denotes the time derivative which is indifferently the material or the partial time derivative as the system is, respectively, in motion or at rest. It is assumed that $\eta$ is a concave function of the variables to guarantee stability of the equilibrium state and that it obeys a 


\section{Nanoscale heat transfer}

general time-evolution equation of the form

$$
\partial_{t} \eta=-\nabla \cdot \mathbf{J}^{p}+\sigma^{p} \quad\left(\sigma^{p}>0\right),
$$

whose rate of production per unit volume $\sigma^{p}$ (in short, the entropy production) is positive definite to satisfy the second principle of thermodynamics, while the quantity $\mathbf{J}^{p}$ is the entropy flux. Let us define the local non-equilibrium temperature by $T^{-1}(e)=\partial \eta / \partial e$ and select the constitutive equation for $\partial \eta / \partial \mathbf{q}$ as given by $\partial \eta / \partial \mathbf{q}=-\gamma(T) \mathbf{q}$, where $\gamma(T)$ is a material coefficient depending generally on $T$; it is positive definite in order to meet the property that $\eta$ is maximum at equilibrium and the minus sign in front of $\gamma(T) \mathbf{q}$ has been introduced for convenience [6]. Under these conditions, Equation (11), referred to as the Gibbs equation, can be written as

$$
d_{t} \eta(e, \mathbf{q})=T^{-1} d_{t} e-\gamma \mathbf{q} \cdot d_{t} \mathbf{q} .
$$

Eliminating $d_{t} e$ by means of the energy balance which, in absence of heat sources, can be written as

$$
d_{t} e=-\nabla \cdot \mathbf{q},
$$

yields

$$
d_{t} \eta=-\nabla \cdot \frac{\mathbf{q}}{T}+\mathbf{q} \cdot\left(\nabla T^{-1}-\gamma d_{t} \mathbf{q}\right) .
$$

From the comparison with the general balance relation (12) follows the identification

$$
\begin{array}{r}
\mathbf{J}^{p}=\mathbf{q} / T \text { (entropy flux) } \\
\sigma^{p}=\mathbf{q} \cdot\left(\nabla T^{-1}-\gamma d_{t} \mathbf{q}\right) \quad \text { (entropy production). }
\end{array}
$$

The expression for $\sigma^{p}$ is a bilinear relationship in the flux $\mathbf{q}$ and the quantity represented by the two terms between the parentheses, which is usually called the thermodynamic force $\mathbf{X}$. The simplest way to guarantee the positiveness of the entropy production $\sigma^{p}$ is to assume a linear flux-force relation of the form $\mathbf{q}=L \mathbf{X}$, where $L$ is a phenomenological coefficient. This procedure leads to Cattaneo's law [12]

$$
\tau d_{t} \mathbf{q}=-\mathbf{q}-\lambda \nabla T,
$$




\section{H. Machrafi}

after one has put $\gamma L=\tau$ (relaxation time) and $L / T^{2}=\lambda$ (heat conductivity) and wherein $\tau$ and $\lambda$ are proven to be positive quantities $[6,11]$. Although Cattaneo's relation is useful at short time scales (high frequencies), it is not satisfactory for the purpose to describe heat transport at short length scales wherein non-localities play a preponderant role. Nonlocal effects are introduced in the framework of EIT by appealing to a hierarchy of fluxes. Let us define these fluxes as $\mathbf{Q}^{(1)}, \mathbf{Q}^{(2)}, \ldots, \mathbf{Q}^{(n)}$ with $\mathbf{Q}^{(1)}$ being identified with the heat flux vector $\mathbf{q}, \mathbf{Q}^{(2)}$ (a tensor of rank two) being the flux of the heat flux, $\mathbf{Q}^{(3)}$ the flux of $\mathbf{Q}^{(2)}$ and so on. From the kinetic theory point of view, the quantities $\mathbf{Q}^{(2)}$ and $\mathbf{Q}^{(3)}$ represent the higher moments of the velocity distribution. Up to the $n^{\text {th }}$-order moment, the Gibbs equation generalizing expression (13) takes the form

$$
\begin{array}{r}
d_{t} \eta\left(e, \mathbf{q}, \mathbf{Q}^{(2)}, \ldots, \mathbf{Q}^{(n)}\right)=T^{-1} d_{t} e-\gamma_{1} \mathbf{q} \cdot d_{t} \mathbf{q}-\gamma_{2} \mathbf{Q}^{(2)} \otimes d_{t} \mathbf{Q}^{(2)}-\ldots \\
-\gamma_{N} \mathbf{Q}^{(N)} \otimes d_{t} \mathbf{Q}^{(N)},
\end{array}
$$

while instead of $\mathbf{J}^{p}=\mathbf{q} / T$, the entropy flux reads as

$$
\mathbf{J}^{p}=T^{-1} \mathbf{q}+\beta_{1} \mathbf{Q}^{(2)} \cdot \mathbf{q}+\ldots+\beta_{N-1} \mathbf{Q}^{(N)} \otimes \mathbf{Q}^{(N-1)},
$$

the symbol $\otimes$ denoting the inner product of the corresponding tensors. For instance, in Cartesian coordinates and using the summation convention on repeated indices, $\mathbf{Q}^{(3)} \otimes \mathbf{Q}^{(2)}$ stands for $Q_{i j k} Q_{j k}$. We have limited ourselves to the simplest form of the entropy and the entropy flux which are sufficient for the present purpose. The entropy production $\sigma^{p}$, which in virtue of (12), is given by

$$
\sigma^{p}=d_{t} \eta+\nabla \cdot \mathbf{J}^{p}
$$

is easily derived by substituting $d_{t} \eta$ and $\mathbf{J}^{p}$ from (18) and (19), respectively, and by eliminating $d_{t} e$ via the energy balance (13), the result is

$$
\begin{array}{r}
\sigma^{p}=-\left(-\nabla T^{-1}+\gamma_{1} d_{t} \mathbf{q}-\beta_{1} \nabla \cdot \mathbf{Q}^{(2)}\right) \cdot \mathbf{q}-\ldots \\
-\sum_{n=2}^{N} \mathbf{Q}^{(n)} \otimes\left(\gamma_{n} d_{t} \mathbf{Q}^{(n)}-\beta_{n} \nabla \cdot \mathbf{Q}^{(n+1)}-\beta_{n-1} \nabla \mathbf{Q}^{(n-1)}\right) \geq 0 .
\end{array}
$$

The above bilinear expression in fluxes and forces (the quantities between parentheses) suggests the following hierarchy of linear flux-force relations

$$
\nabla T^{-1}-\gamma_{1} d_{t} \mathbf{q}+\beta_{1} \nabla \cdot \mathbf{Q}^{(2)}=\mu_{1} \mathbf{q}
$$




\section{Nanoscale heat transfer}

(23) $\beta_{n-1} \nabla \mathbf{Q}^{(n-1)}-\gamma_{n} d_{t} \mathbf{Q}^{(n)}+\beta_{n} \nabla \cdot \mathbf{Q}^{(n+1)}=\mu_{n} \mathbf{Q}^{(n)}, \quad(\mathrm{n}=2,3, \ldots, \mathrm{N})$.

The latter can also be viewed as time evolution equations for the fluxes $\mathbf{q}$, $\mathbf{Q}^{(2)}, \ldots, \mathbf{Q}^{(N)}$. Making use of Equations (22) and (23), Equation (21) of the entropy production becomes

$$
\sigma^{p}=\mu_{1} \mathbf{q} \cdot \mathbf{q}+\mu_{2} \mathbf{Q}^{(2)} \otimes \mathbf{Q}^{(2)}+\ldots+\mu_{N} \mathbf{Q}^{(N)} \otimes \mathbf{Q}^{(N)} \geq 0,
$$

with $\mu_{n} \geq 0(n=1,2, \ldots, N)$ to satisfy the positiveness of the entropy production.

\subsection{Mathematical model for the thermal conductivities}

Before developing the nanoparticle thermal conductivity, we start with the matrix. For the heat conductivity of the matrix, we use the classical Boltzmann phonon expression

$$
\lambda_{m}^{s}=\left.\frac{1}{3}\left(C_{m} v_{m} \Lambda_{m}^{s}\right)\right|_{T_{r e f}},
$$

$$
\lambda_{m}^{c}=\left.\frac{1}{3}\left(C_{m} v_{m} \Lambda_{m}^{c}\right)\right|_{T_{r e f}}
$$

$T_{r e f}$ is the reference temperature, say the room temperature, and $\Lambda_{m}^{s}$ and $\Lambda_{m}^{s}$ are the phonon mean free paths of the matrix in the case of spherical and cylindrical nanoparticles, respectively. Within the matrix, the phonons experience phonon-phonon interactions and the mean free path (for both types of shapes) is given by the Matthiessen rule:

$$
\frac{1}{\Lambda_{m}}=\frac{1}{\Lambda_{m, b}}+\frac{1}{\Lambda_{m, \text { coll }}},
$$

with $\Lambda_{m, b}$ designating the mean free path in the bulk (the same whatever the shape of the nanoparticle) and $\Lambda_{m \text {,coll }}$ the supplementary contribution due to the interactions at the particle-matrix interface given by [9]

$$
\Lambda_{m, \text { coll }}^{s}=4 r_{p, s} / 3 \varphi
$$




\section{H. Machrafi}

in the case of spherical nanoparticles and by [13]

$$
\Lambda_{m, \text { coll }}^{c}=\frac{1+s}{1-s} \frac{1}{\varphi\left(\frac{2}{\pi r_{p}} \sin \theta+\left(\frac{1}{L}+\frac{2 \zeta}{\pi r_{p}}\right) \cos \theta\right)},
$$

in the case of cylindrical nanoparticles. Here, $\zeta=\sqrt{\varphi} /(\sqrt{\varphi}+1)$. If $\theta=0$, the heat direction would be in the longitudinal direction. If $\theta=\pi / 2$, the heat direction would be radial. In this work, we choose a mean value of $\theta=\pi / 4$ [13], including therefore both the longitudinal and radial contributions. Concerning the derivation of $\lambda_{p}$ (for both spherical and cylindrical nanoparticles), we propose a new closed-form formula

$$
\lambda_{p}=\lambda_{p}^{0} f(K n, s),
$$

wherein $\lambda_{p}^{0}$ is the value of the thermal conductivity for the bulk material of which the particle is composed of and given by an expression similar to Equations (25) and (26):

$$
\lambda_{p}^{0}=\left.\frac{1}{3}\left(C_{p} v_{p} \Lambda_{p, b}\right)\right|_{T_{r e f}},
$$

where the quantity $f(K n, s)$ is a correction factor, taking into account the dimension of the nanoparticles (via the Knudsen number $K n$ ), their shape (spherical or cylindrical) and their specularity (via the specularity coefficient $s)$. Note that $\lambda_{p}^{0}$ is the same, whether it be in the spherical case $\left(\lambda_{p}^{s, 0}\right)$ or in the cylindrical case $\left(\lambda_{p}^{c, 0}\right)$. The Knudsen number is defined as

$$
K n=\Lambda_{p, b} / \ell_{p, s}
$$

Expression (31) is analogous to that used for the matrix with the exception that now the mean free path is only the bulk one (so that $\Lambda_{p}=\Lambda_{p, b}$ and thus equal for both the sperical and cylindrical cases). The contribution of the collisions are hidden in the correction factor $f(K n)$. This correction factor can be determined by continuing the development from Equations (22) and (23). There $\gamma_{i}, \beta_{i}$ and $\mu_{i}$ are phenomenological coefficients related to the relaxation times, correlation lengths and transport coefficients, respectively. In order to gain insight about the physical meaning of these phenomenological coefficients, let us assume absence of non-locality so that the term in $\nabla \cdot \mathbf{Q}^{(2)}$ will not appear in Equation (22) which reduces to Cattaneo's relation (see Equation (17)) [12]. If, in addition, one considers steady 


\section{Nanoscale heat transfer}

situations, the term in $d_{t} \mathbf{q}$ vanishes and one recovers Fourier's law. These observations lead to the following identities

$$
\begin{aligned}
& \mu_{1}=\frac{1}{\lambda T^{2}}, \\
& \gamma_{1}=\frac{\tau}{\lambda T^{2}},
\end{aligned}
$$

indicating that $\mu_{1}$ is related to the heat conductivity $\lambda$ and $\gamma_{1}$ to the relaxation time $\tau$. The identification of the higher order coefficients is not so easy as its demands to compare with higher order evolution equations, but the parameters $\mu_{n}$ and $\gamma_{n}$ are related to coefficients of thermal conductivity $\lambda_{n}$ and relaxation times $\tau_{n}$ of order $n$, respectively. Moreover, since $\mathbf{Q}^{(n+1)}$ is the flux of $\mathbf{Q}^{(n)}$, this implies, by the very definition of a flux, that $d_{t} \mathbf{Q}^{(n)}=-\nabla \cdot \mathbf{Q}^{(n+1)}$. Well, when dividing Equation (22) by $\gamma_{1}$ and Equation (23) by $\gamma_{n}(n=2,3, \ldots)$, it follows that $\beta_{1} / \gamma_{1}=-1, \beta_{2} / \gamma_{2}=-1$ or, more generally, $\gamma_{n}=-\beta_{n}$, which reduces considerably the number of undetermined coefficients.

Let us now consider an infinite number of flux variables $(N \rightarrow \infty)$ and apply the spatial Fourier transform

$$
\widehat{\mathbf{q}}(\mathbf{k}, t)=\int_{-\infty}^{+\infty} \mathbf{q}(\mathbf{r}, t) e^{-i \mathbf{k} \cdot \mathbf{r}} d \mathbf{r}
$$

to Equations (22) and (23), with $\widehat{\mathbf{q}}$ the Fourier transform of $\mathbf{q}, \mathbf{r}$ the spatial variable, $t$ the time and $\mathbf{k}$ the wavenumber vector. This procedure results into obtaining the following time-evolution equation for the heat flux:

$$
\bar{\tau}(\mathbf{k}) \partial_{t} \widehat{\mathbf{q}}(\mathbf{k}, t)+\widehat{\mathbf{q}}(\mathbf{k}, t)=-i \mathbf{k} \lambda_{p}(\mathbf{k}) \widehat{T}(\mathbf{k}, t),
$$

where $\bar{\tau}(\mathbf{k})=\gamma_{1} / \mu_{1}$ designates a renormalized relaxation time depending generally on $\mathbf{k} . \lambda_{p}(\mathbf{k})$ is given by the continued-fraction for the $\mathbf{k}$-dependent effective thermal conductivity:

$$
\lambda_{p}(\mathbf{k})=\frac{\lambda_{p}^{0}}{1+\frac{\mathbf{k}^{2} l_{1}^{2}}{1+\frac{\mathbf{k}^{2} l_{2}^{2}}{1+\mathbf{k}^{2} l_{3}^{2}}}},
$$




\section{H. Machrafi}

with $\lambda_{p}^{0}$ the classical bulk thermal conductivity, given by Equation (31) (independent of the dimension and shape of the system) $l_{n}$ is the correlation length of order $n$ defined by $l_{n}^{2}=\beta_{n}^{2} /\left(\mu_{n} \mu_{n+1}\right)$. Here, it is assumed that the relaxation times $\tau_{n}(n>1)$ corresponding to higher order fluxes are negligible with respect to $\tau_{1}$, which is a hypothesis generally admitted in kinetic theories.

Now, in the case of spherical nanoparticles, there is only one dimension, namely the specular radius $r_{p}$ of the spheres, so that it is natural to define the wavenumber (now a scalar) $k \equiv 2 \pi / \ell_{p, s}$, with $\ell_{p}=r_{p}$. The correlation lengths selected as $l_{n}^{2}=a_{n+1} l^{2}$, with $a_{n}=n^{2} /\left(4 n^{2}-1\right)$ and $l$ identified as the mean free path independently of the order of approximation. This is a rather natural choice for phonons as shown by Dreyer and Struchtrup [14]. With these results in mind and the definition of the Knudsen number (32), the continued fraction (37) reduces to an asymptotic limit (the mathematical description is given in [15]), leading finally to the following expression for the thermal conductivity of spherical nanoparticles $\lambda_{p}^{s}$ :

$$
\lambda_{p}^{s}=\frac{3 \lambda_{p}^{0}}{4 \pi^{2} K n^{2}}\left(\frac{2 \pi K n}{\arctan (2 \pi K n)}-1\right),
$$

so that $f(K n, s)=\frac{3}{4 \pi^{2} K n^{2}}\left(\frac{2 \pi K n}{\arctan (2 \pi K n)}-1\right)$.

In case of cylindrical nanoparticles, there are two dimensions, namely the radial dimension $r_{p}$ and the longitudinal one $L$ (length of the cylindrical nanoparticle). Following [6,16], all the $l_{n}$ 's are equal $l_{n}=\Lambda_{p, b} / 2$ with $\Lambda_{p, b}$ the bulk mean free path of the phonons of the particles. By identifying $\mathbf{k}$ as (becoming again a scalar now) $k=2 \pi / \ell_{p, s}$ (with $\ell_{p}$ now given by Equation (10)) and the definition of the Knudsen number (32), expression (37) has now the asymptotic limit

$$
\lambda_{p}^{c}=\frac{\lambda_{p}^{0}}{2 \pi^{2} K n^{2}}\left(\sqrt{1+4 \pi^{2} K n^{2}}-1\right) .
$$

so that now $f(K n, s)=\frac{1}{2 \pi^{2} K n^{2}}\left(\sqrt{1+4 \pi^{2} K n^{2}}-1\right)$.

Having now established the mathematical model, we proceed with applying it to a copper-silicon nanocomposite in order to assess various nanometric effects on the effective thermal conductivity.

\section{Application to Copper-Silicon nanocomposites}

\subsection{Physical properties}

The mathematical model described in this work is applied to a coppersilicium $(\mathrm{Cu}-\mathrm{Si})$ nanocomposite, with $\mathrm{Cu}$ nanoparticles embedded in a $\mathrm{Si}$ 


\section{Nanoscale heat transfer}

host matrix. Table 1 presents the physical properties of the considered system.

Table 1. Phonon properties for bulk materials at room temperature $\left(T_{\text {ref }}\right)$.

\begin{tabular}{c|c|c|c} 
Material & Heat capacity $\left[\mathrm{MJm}^{-3} K\right]$ & Group velocity $[\mathrm{m} / \mathrm{s}]$ & Mean free path $[\mathrm{nm}]$ \\
\hline $\mathrm{Si}$ & $0.93^{a}$ & $1804^{a}$ & $268^{a}$ \\
$\mathrm{Cu}$ & $3.47^{b}$ & $7723^{c}$ & $45^{d}$ \\
\hline
\end{tabular}

${ }^{a}[13]$

$b[17]$

${ }^{c}$ Calculated from $v=3 \lambda / \Lambda_{b}$ by considering $\lambda=402 \mathrm{Wm}^{-1} \mathrm{~K}^{-1}$ from [18] $d$ [18]

\subsection{Comparison to other models}

We compare our model to two other models (one taking into account the effect of phonon scattering and the other not), in order to highlight some important aspects concerning our model, before discussing some parameters that effect the thermal conductivity. Since the models with which we present ours concerns spherical nanoparticles, we only discuss the latter ones in this subsection. Figure 1 presents the effective thermal conductivity versus the volume fraction of spherical $\mathrm{Cu}$ nanoparticles embedded in a Si host matrix. The results of two other models are also included. One is an analytical model based on Bruggeman's model adapted for high volume fractions [18]. The second is based on Fourier's law [8]. The comparison is performed for four different particle radii: 50, 500, 900 and $3000 \mathrm{~nm}$. For the moment, we consider zero specularity $(s=0)$.

First, it is interesting to notice from the results of our model that, at $r_{p}=50$ $\mathrm{nm}$, a higher volume fraction of $\mathrm{Cu}$ nanoparticles decrease considerably the effective thermal conductivity, knowing that $\mathrm{Cu}$ has a much higher bulk thermal conductivity than Si. This shows the strong influence of phonon scattering on heat transfer at nanometric scales. As the particle radius increases, we can see that for a given volume fraction the effective thermal conductivity increases. This means that the scattering effect decreases. Comparing our model with the one in [8], we can see that eventually at $r_{p}=3000 \mathrm{~nm}$ ours coincides with the latter. Indeed, we can say for the present system that at micrometric scales the scattering effect is hardly present, leading our model to correspond to Fourier's limit. For smaller particle radii, our model differs considerably from the one in [8], from which can be concluded that the validity of Fourier's law is clearly questionable at small length scales and that phonon scattering is an important phenomenon to be taken into account at nanoscale. This is confirmed by the satisfactory 


\section{H. Machrafi}

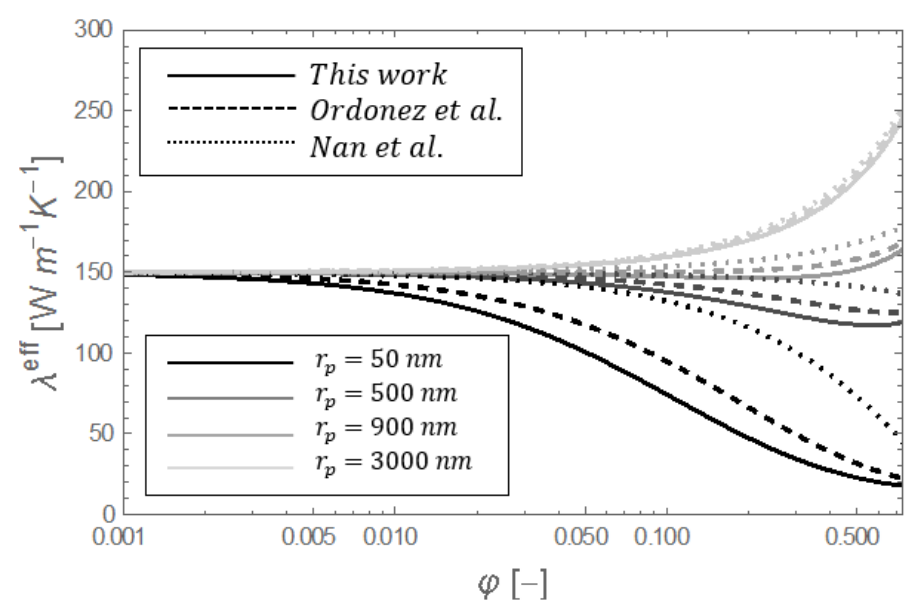

Figure 1. Effective thermal conductivity $\left(\lambda^{e f f}\right)$ of the spherical nanocomposite Cu-Si as a function of the volume fraction $(\varphi)$ of $\mathrm{Cu}$ nanoparticles for different particle radii: $r_{p}=50,500,900$ and $3000 \mathrm{~nm}(s=0)$. Comparison with theoretical models (see [8] and $[18])$.

comparison with [18], which also takes into account the scattering effect at nanoscale. Finally, we can say that by controlling the particle size, one may control increase or decrease of the effective thermal conductivity.

\subsection{Effect of particle size on the effective thermal conductivity}

We have seen in Section 3.2 that the size of the particles play a crucial role. Therefore, Figure 2 presents the effective thermal conductivity versus the inverse Knudsen number for spherical particles (with zero specularity) $\left.K n^{-1}=r_{p} / \Lambda_{p, b}\right)$. For information, the two other models considered in this work [18] and [8] are included.

The minimum value of $K n^{-1}$ in Figure 2 is 0.01 . This corresponds to $r_{p}=4.5 \AA$, which is the size of a few copper atoms, which we consider a limiting value for a nanoparticle radius. Therefore, smaller $K n^{-1}$-values are not presented. Nevertheless, Figure 2 shows two clear limits for the effective thermal conductivity with respect to the particle size, here dimensionlessly indicated by the inverse Knudsen number $\mathrm{Kn}^{-1}$. Figure 2 shows that for a decreasing nanoparticle size (lower $K n^{-1}$-values), the effective thermal conductivity decreases. Smaller nanoparticle sizes (for a given volume fraction) result into a larger total particle-matrix surface, which forms more obstacles for the colliding phonons. Also, the thermal boundary resistance increases (see Equation (5) and also Equation (6) for the cylindrical case). This causes a reduction in heat transfer and consequently the effective ther- 


\section{Nanoscale heat transfer}

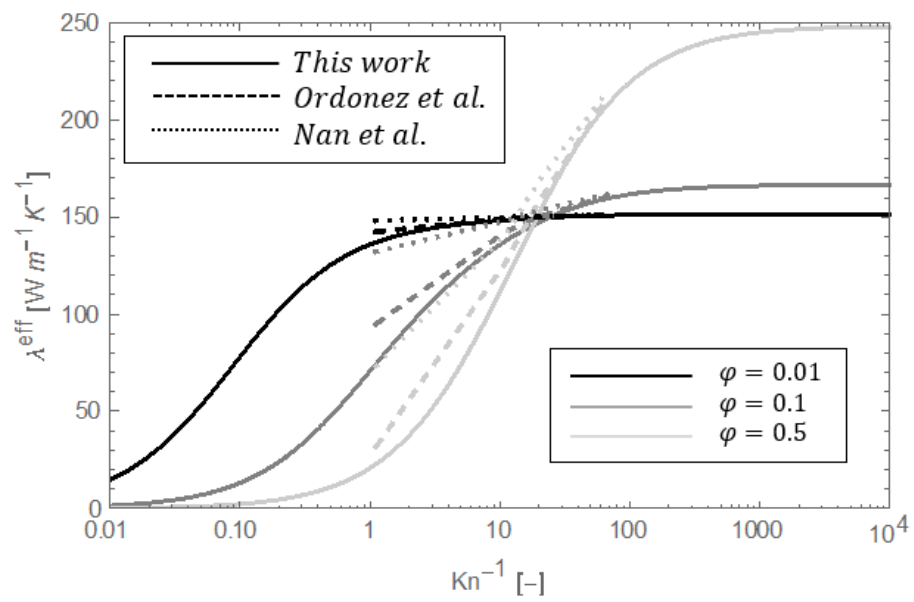

Figure 2. Effective thermal conductivity $\left(\lambda^{\text {eff }}\right)$ of the spherical nanocomposite $\mathrm{Cu}-\mathrm{Si}$ as a function of the inverse Knudsen number $\left(\mathrm{Kn}^{-1}\right)$ for different $\mathrm{Cu}$ nanoparticle volume fractions: $\varphi=0.01,0.1$ and $0.5(s=0)$. The models [8] and [18] are also added for information.

mal conductivity. In the mathematical limit of $K n^{-1} \rightarrow 0$ or $K n \rightarrow \infty$ (and thus $r_{p} \rightarrow 0$ ), it can be easily verified that $\lambda_{p}^{s} \rightarrow 0, \lambda_{m}^{s} \rightarrow 0$ and therefore $\lambda^{e f f, s} \rightarrow 0$, which can be seen in Figure 1. As $\mathrm{Kn}^{-1}$ increases, we can see that, apparently, an asymptotic maximum value for the effective thermal conductivity exists with respect to the inverse Knudsen number as $K n \rightarrow 0$ and $r_{p} \rightarrow \infty$, for whatever volume fraction. We can find this value analytically by stating that for spherical nanoparticles

$$
\left.\lambda_{p}^{s}\right|_{K n \rightarrow 0}=\lambda_{p}^{0}
$$

$$
\left.\alpha^{s}\right|_{r_{p} \rightarrow \infty}=0
$$

$$
\left.\Lambda_{m}^{s}\right|_{r_{p} \rightarrow \infty}=\Lambda_{m, b}
$$

$$
\left.\lambda_{m}^{s}\right|_{r_{p} \rightarrow \infty}=\lambda_{m}^{0}
$$

where $\lambda_{m}^{0}$ is the basic material thermal conductivity of the matrix without 


\section{H. Machrafi}

embedded particles. This leads finally to

$$
\lambda^{e f f, s, 0}(\varphi)=\left.\lambda^{e f f, s}\right|_{K n \rightarrow 0}=\lambda_{m}^{0}\left(1+\frac{3 \varphi\left(\lambda_{p}^{0}-\lambda_{m}^{0}\right)}{2 \lambda_{m}^{0}+\lambda_{p}^{0}-\varphi\left(\lambda_{p}^{0}-\lambda_{m}^{0}\right)}\right),
$$

which is only dependent on the volume fraction, knowing the basic material thermal conductivities. From Table 1, we can find $\lambda_{p}^{0}=402 \mathrm{Wm}^{-1} \mathrm{~K}^{-1}$ and $\lambda_{m}^{0}=150 \mathrm{Wm}^{-1} \mathrm{~K}^{-1}$. This allows finding $\lambda^{\text {eff }, s, 0}(0.01) \approx 151 \mathrm{Wm}^{-1} \mathrm{~K}^{-1}$, $\lambda^{e f f, s, 0}(0.1) \approx 167 \mathrm{Wm}^{-1} \mathrm{~K}^{-1}$ and $\lambda^{\text {eff }, s, 0}(0.5) \approx 248 \mathrm{Wm}^{-1} \mathrm{~K}^{-1}$, which corresponds with the asymptotic values (for $\mathrm{Kn}^{-1} \rightarrow \infty$ ) in Figure 2 .

Figure 3 is the equivalent of Figure 2, but for the cylindrical case (presenting only our model), with $L \rightarrow \infty$.

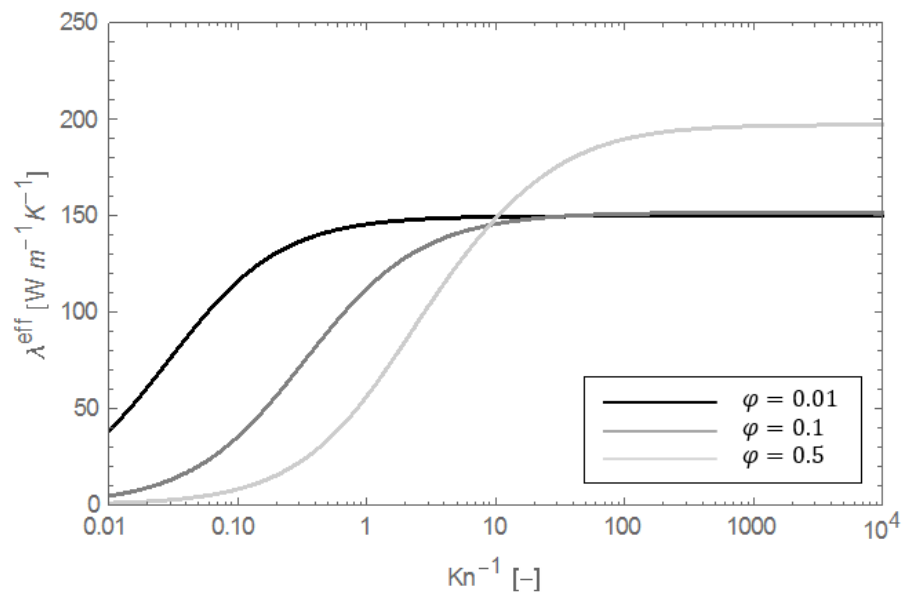

Figure 3. Effective thermal conductivity $\left(\lambda^{\text {eff }}\right)$ of the cylindrical nanocomposite $\mathrm{Cu}-\mathrm{Si}$ as a function of the inverse Knudsen number $\left(K^{-1}\right)$ for different $\mathrm{Cu}$ nanoparticle volume fractions: $\varphi=0.01,0.1$ and $0.5(s=0$ and $L \rightarrow \infty)$.

The same observations and conclusions can be drawn from Figure 3 as from Figure 2. Performing the same asymptotic study as for the spherical case, it is for the cylindrical case easy to verify that for $K n \rightarrow \infty, \lambda^{e f f, c} \rightarrow 0$. As for the limit $K n \rightarrow 0$, it is also easy to verify that for $L \rightarrow \infty$

$$
\left.\lambda_{p}^{c}\right|_{K n \rightarrow 0}=\lambda_{p}^{0}
$$

$$
\left.\alpha^{c}\right|_{r_{p} \rightarrow \infty}=0
$$




$$
\left.\Lambda_{m}^{c}\right|_{r_{p} \rightarrow \infty}=\Lambda_{m, b}
$$

$$
\left.\lambda_{m}^{c}\right|_{r_{p} \rightarrow \infty}=\lambda_{m}^{0}
$$

For $L \rightarrow \infty$, this leads finally to

$$
\begin{gathered}
\lambda_{r}^{e f f, c, 0}(\varphi)=\left.\lambda_{r}^{e f f, c}\right|_{K n \rightarrow 0}=\lambda_{m}^{0}\left(1+\frac{2 \varphi\left(\lambda_{p}^{0}-\lambda_{m}^{0}\right)}{\lambda_{m}^{0}+\lambda_{p}^{0}-\varphi\left(\lambda_{p}^{0}-\lambda_{m}^{0}\right)}\right) \\
\lambda_{L}^{e f f, c, 0}(\varphi)=\left.\lambda_{L}^{e f f, c}\right|_{K n \rightarrow 0}=(1-\varphi) \lambda_{m}^{0}+\varphi \lambda_{p}^{0}
\end{gathered}
$$

$$
\lambda^{e f f, c, 0}(\varphi)=\left.\lambda^{e f f, c}\right|_{K n \rightarrow 0}=\frac{\left(3-3 \varphi^{2}\right)\left(\lambda_{m}^{0}\right)^{2}+\left(3+2 \varphi^{2}\right) \lambda_{m}^{0} \lambda_{p}^{0}+\varphi^{2}\left(\lambda_{p}^{0}\right)^{2}}{\left(3+2 \varphi^{2}\right) \lambda_{m}^{0}+\left(3-2 \varphi^{2}\right) \lambda_{p}^{0}}
$$

which is also only dependent on the volume fraction, knowing the basic material thermal conductivities. Again, using the values of $\lambda_{p}^{0}=402$ $W m^{-1} K^{-1}$ and $\lambda_{m}^{0}=150 W^{-1} K^{-1}$, we can find $\lambda^{e f f, c, o}(0.01) \approx$ $150 \mathrm{Wm}^{-1} \mathrm{~K}^{-1}, \lambda^{\text {eff }, c, 0}(0.1) \approx 152 \mathrm{Wm}^{-1} \mathrm{~K}^{-1}$ and $\lambda^{\text {eff }, c, 0}(0.5) \approx 197$ $W m^{-1} K^{-1}$. This corresponds well with the asymptotic values (for $\mathrm{Kn}^{-1} \rightarrow$ $\infty)$ in Figure 3.

\subsection{Effect of the particle shape on the effective thermal conductivity}

We have seen that embedding spherical or cylindrical (with $L>>r_{p}$ ) nanoparticles has a clear influence on the value of the effective thermal conductivity. The goal in this subsection is to asses the difference as a function of the length of the cylindrical nanoparticle, $L$. Figure 4 shows the effective thermal conductivity versus the $\mathrm{Cu}$ volume fraction in a Si matrix for four different values of $L$ : one smaller than the $\mathrm{Cu}$ bulk mean free path $\Lambda_{p, b}, L=10 \mathrm{~nm}$, one equal to the $\mathrm{Cu}$ mean free path, $L=\Lambda_{p, b}$, one sligthly larger, $L=100 \mathrm{~nm}$ and finally $L \rightarrow \infty$. For each $L$-case, the results for the spherical nanoparticles are added for reference.

We can see from Figure 4 that the cylinder length influences greatly the effective thermal conductivity for this system. At $L=10 \mathrm{~nm}, L$ is so small that the $L$-contribution in the collisional mean free path is larger than the 


\section{H. Machrafi}
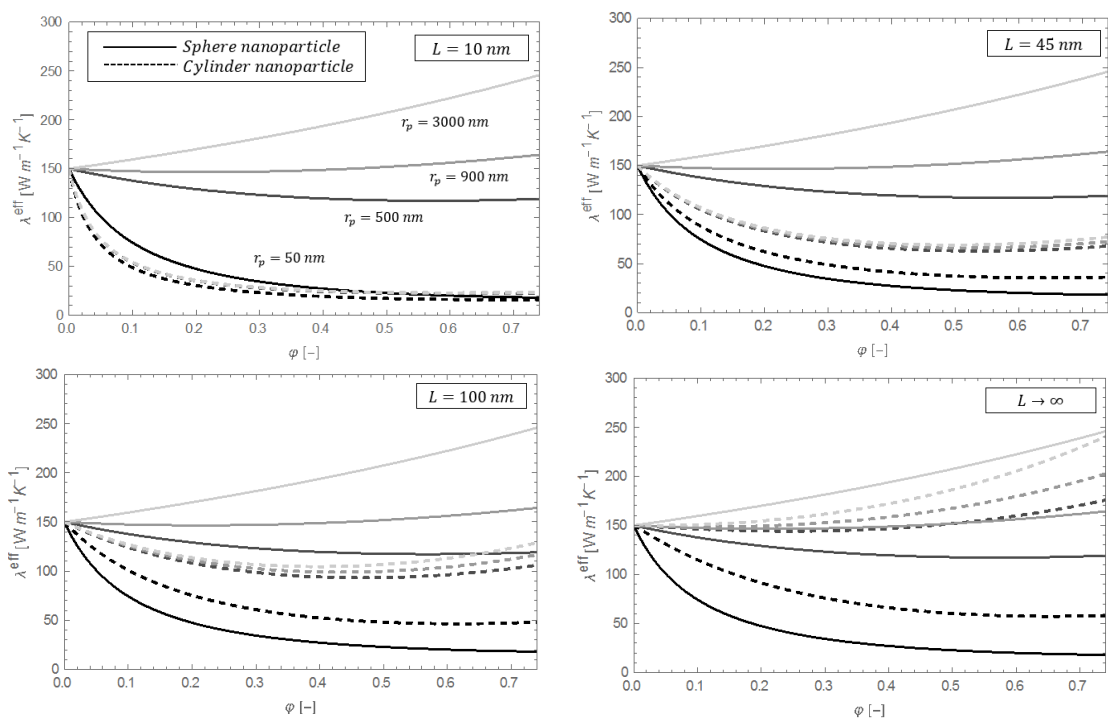

Figure 4. Effective thermal conductivity $\left(\lambda^{\text {eff }}\right)$ of the cylindrical nanocomposite $\mathrm{Cu}-\mathrm{Si}$ as a function of the volume fraction $(\varphi)$ for different $\mathrm{Cu}$ nanoparticle cylinder lengths: $L=10,45,100 \mathrm{~nm}$ and $L \rightarrow \infty(s=0)$.

$r_{p}$-contribution (see Equation (29)), so that, whatever the value of $r_{p}$, the scattering effect is quite high. This causes the effective thermal conductivity to decreases considerably, with hardly any influence of $r_{p}$. For $L=45$ $\mathrm{nm}$, the effective thermal conductivity becomes comparable to that of the spherical case at $r_{p}=50 \mathrm{~nm}$, but still much smaller for the other $r_{p}$-values. As $L$ increases, we can see that at $L=100 \mathrm{~nm}$, the effective thermal conductivities in the cylindrical case keep on increasing. This effect is stronger for larger $r_{p}$ values. This can be understood, by noticing that for smaller $r_{p}$-values, the scattering effect is caused by the radial contribution. Indeed, this shows that the scattering effect is caused by both the radial and longitudinal contributions. Finally, as $L \rightarrow \infty$, the effective thermal conductivity reaches its maximum value for a given $r_{p}$-value. It is should be noted that this is not equal to the absolute maximum value of the effective thermal conductivity (when both $r_{p} \rightarrow \infty$ and $L \rightarrow \infty$, which is well approached in the case $r_{p}=3000 \mathrm{~nm}$ ), which is, for the cylindrical case, given by $\lambda^{e f f, c, 0}(\varphi)$ in Equation (51).

\subsection{Effect of the specularity parameter on the effective thermal conductivity}

Up until now, we have considered $s=0$. In this subsection, we assess the influence of this parameter on the effective thermal conductivity of spherical $\mathrm{Cu}$ nanoparticles in $\mathrm{Si}$ (since the effect of $s$ on the cylindrical case is the 


\section{Nanoscale heat transfer}

same, we do not present the results here). Figure 5 presents the equivalence of Figure 1, considering four cases of the specularity: $s=0,0.2,0.5$ and $s \rightarrow 1$. For comparison, the models of [18] and [8] are included.
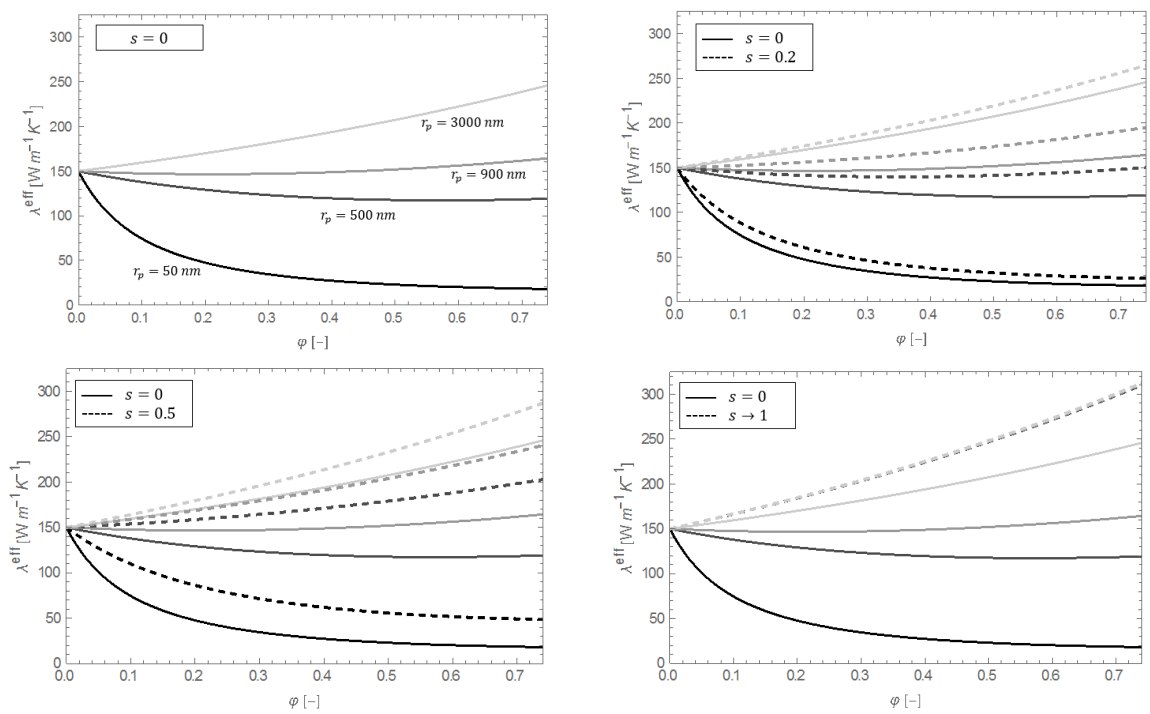

Figure 5. Effective thermal conductivity $\left(\lambda^{\text {eff }}\right)$ of the cylindrical nanocomposite $\mathrm{Cu}-\mathrm{Si}$ as a function of the volume fraction $(\varphi)$ for different specularities $s=0,0.2,0.5$ and 1 .

Figure 5 also shows that our model with $s=0.2$ does not differ much from the curves with $s=0$. For $s>0.2$, the difference becomes considerable. Knowing that our model with $s=0$ coincided well with the one in [18] and that our model with $s=0.2$ also does (since it does not differ much from the curves with $s=0$ ) implies that for our system the nanoparticle surface is such that $0<s<0.2$. We can see that, generally, increasing the specularity factor increases the effective thermal conductivity. A nanoparticle with a larger specularity means that the scattering effect is less (as if the nanoparticle surface is smoother towards incoming phonons). So, less scattering induces less obstacles for the phonons, which increases the effective thermal conductivity until no scattering $s \rightarrow 1$. Note that the curves in Figure 5 for $s \rightarrow 1$ have approximately the same value so that they are hardly distinguishable. This can be understood by recalling that $s \rightarrow 1$ also means $r_{p, s} \rightarrow \infty$ (so that the value of $r_{p}$ does hardly matter) and $K n \rightarrow 0$. This observation means also that no scattering $(s \rightarrow 1)$ corresponds to obtaining the maximal effective thermal conductivity for the spherical case $\lambda^{e f f, s, O}(\varphi)$, given by Equation (44). 


\section{H. Machrafi}

\section{Acknowledgements}

The author cordially acknowledges Prof. Georgy Lebon for valuable discussions. Financial support from BelSPo is also acknowledged.

\section{REFERENCES}

1. D. C. Moreira, L. A. Sphaier, J. M. L. Reis, and L. C. S. Nunes, Experimental investigation of heat conduction in polyester $\mathrm{Al} 2 \mathrm{O} 3$ and polyester CuO nanocomposites, Journal of Experimental Fluid Science, vol. 35, pp. 1458-1462, 2011.

2. J. Chen, S. L. Li, Z. L. Tao, and L. Z. Zhang, Reversible hydrogen and lithium storage of mos2 nanotubes, International Journal of Nanoscience, vol. 1, pp. 295-302, 2002.

3. M. Wang, N. Pan, J. Wang, and S. Chen, Mesoscopic simulations of phase distribution effects on the effective thermal conductivity of microgranular porous media, Journal of Colloid and Interface Science, vol. 311, pp. 562-570, 2007.

4. M. Wang, X. Wang, J. Wang, and N. Pan, Grain size effects on effective thermal conductivity of porous materials with internal thermal contact resistance, Journal of Porous Media, vol. 16, pp. 1043-1048, 2013.

5. D. Jou, A. Sellitto, and V. A. Cimmelli, Multi-temperature mixture of phonons and electrons and nonlocal thermoelectric transport in thin layers, International Journal of Heat and Mass Transfer, vol. 71, pp. 459$468,2014$.

6. D. Jou, J. Casas-Vazquez, and G. Lebon, Extended Irreversible Thermodynamics. Springer-Verlag, 2010.

7. G. Lebon, H. Machrafi, M. Grmela, and C. Dubois, An extended thermodynamic model of transient heat conduction at sub-continuum scales, Proceedings of the Royal Society A, vol. 467, pp. 3241-3256, 2011.

8. C. Nan, R. Birringer, D. R. Clarke, and H. Gleiter, Effective thermal conductivity of particulate composites with interfacial thermal resistance, Journal of Applied Physics, vol. 81, pp. 6692-6699, 1997.

9. A. Minnich and G. Chen, Modified effective medium formulation for the thermal conductivity of nanocomposites, Applied Physics Letters, vol. 91, p. 073105, 2007.

10. G. Chen, Thermal conductivity and ballistic-phonon transport in the cross-plane direction of superlattices, Physics Review B, vol. 57, pp. 14958-14973, 1998.

11. D. Jou, J. Casas-Vazquez, and G. Lebon, Understanding NonEquilibrium Thermodynamics. Springer-Verlag, 2008.

12. C. Cattaneo, Sulla conduzione, in Atti del Seminario Matematico e 


\section{Nanoscale heat transfer}

Fisico delle Universita di Modena, vol. 3, pp. 83-101, 1948.

13. A. Behrang, M. Grmela, C. Dubois, S. Turenne, P. G. Lafleur, and G. Lebon, Effective heat conduction in dispersion of wires, Applied Physics Letters, vol. 104, p. 063106, 2014.

14. W. Dreyer and H. Struchtrup, Heat pulse experiments revisited, Continuum Mechanics Thermodynamics, vol. 3, pp. 3-50, 2003.

15. S. Hess, On nonlocal constitutive relations, continued fraction expansion for the wave vector dependent diffusion coefficient, Zeitschrift Naturforschung, vol. 32a, pp. 678-684, 1977.

16. D. Jou, J. Casas-Vazquez, G. Lebon, and M. Grmela, A phenomenological scaling approach for heat transport in nano-systems, Applied Mathematics Letters, vol. 18, pp. 963-967, 2005.

17. R. H. Perry, D. W. Green, and J. O. Maloney, Perry's Chemical Engineers' Handbook. McGraw-Hill, 1997.

18. J. Ordonez-Miranda and J. J. Alvarado-Gil, Thermal conductivity of nano-composites with high volume fractions of particles, Composites Science and Technology, vol. 72, pp. 853-857, 2012. 